\title{
Non-motor symptoms in Parkinson's disease
}

\author{
Manifestações não motoras na doença de Parkinson
}

Egberto Reis Barbosa

\author{
Livre-Docente do Departamento de \\ Neurologia da Faculdade de Medicina \\ da Universidade de São Paulo \\ (FMUSP), São Paulo SP, Brazil.

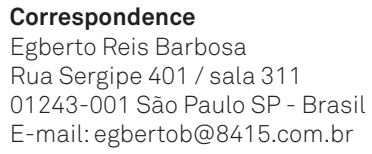 \\ Conflict of interest \\ There is no conflict of interest to \\ declare. \\ Received 16 February 2013 \\ Accepted 22 February 2013
}

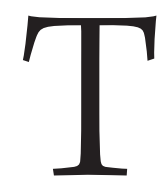

he classic features of the clinical picture in Parkinson's disease (PD) are the motor disorders: akinesia, rigidity, tremor and postural instability. However, non-motor symptoms (NMS) are frequently present. Therefore, patients with PD can present, besides the typical parkinsonian motor syndrome, a wide arrow of NMS, mainly neuropsychiatric manifestations (as depression and cognitive impairment), sleep disorders (as rapid eye movement behavior disorder), autonomic dysfunctions (as intestinal constipation, postural hypotension and miccional disorders) and others, as fatigue and pain. In their various combinations, NMS may eventually become the main complaints of patients with PD.

NMS may be related to the neurodegenerative changes affecting several neural systems and/or caused by drugs employed in the treatment of PD. The mainly neural systems related to NMS are: non-motor frontostriatal circuits, serotoninergic nuclei of median raphe, noradrenergic nuclei (locus coeruleus and subcoeruleus), autonomic centers (hypothalamus and dorsal motor of vagus) and olfactory system.

The clinical picture of PD has been compared with an iceberg. The visible part represents the motor symptoms, and the larger non-visible part represents the several NMS, often underecognized in clinical practice.

The study of Cosentino et al..$^{1}$ published in this issue of Arquivos de Neuropsiquiatria was performed aiming to determine the prevalence of NMS in 300 PD outpatients seen at the Movement Disorders Unit of the Instituto Nacional de Ciencias Neurológicas in Lima, Peru. The patients completed the Spanish validated version of the Non-motor Symptoms Questionnaire (NMSQuest). This questionnaire was developed by Chaudhuri et al. ${ }^{2}$ to address the need for a quantitative and validated instrument to measure NMS in PD. The NMSQuest is a simple self-completed tool and it is comprised of 30 items grouped according to 9 NMS domains.

The results of this study show the high prevalence and the variety of NMS in PD: at least one NMS was present in $99.3 \%$ of the patients evaluated and the mean total NMS (NMSQ-T) was 12.41 ranging from 0 to 27 of a maximum of 30 . These data confirm those from previous similar studies. Martinez-Martin et al. ${ }^{3}$ performed a multicentric, international, cross-sectional study involving 545 PD patients (mean disease duration: 6.96 year) using the same questionnaire (NMSQuest) and reported 10.3 as the mean total NMS, and such symptoms were present in $98.4 \%$ of the patients evaluated.

The PRIAMO study (Barone et al. ${ }^{4}$ ) is another important one to be compared to the Cosentino et al. ${ }^{1}$ study. This Italian multicenter survey using a semi-structured interview in 1,072 consecutive patients with PD was performed to assess the prevalence NMS in 12 domains, their association with cognitive impairment and the impact on patients' quality of life. They found that $98.6 \%$ of patients with PD reported the presence of NMS and the mean number of NMS per patient was 7.8 ranging from 0 to 32 .

The NMS in PD may be present in all the clinical phases of the disease: pre-motor phase, the initial phase (when the motor symptoms appear and the diagnosis is made), the moderate phase and in the advanced phase. In the pre-motor phase, the most common NMS symptoms are: hyposmia, depression, intestinal constipation and rapid eye movement behavior disorder. Along the initial and moderate phases, several autonomic and neuropsychiatric manifestations may be present, and in the advanced one the most important NMS is dementia of PD. Consentino et al. ${ }^{1}$ observed that the mean total NMS increased significantly with disease duration. Mean total NMS was 10.74 in patients with less than 5 years of disease, 13.71 between 5 and 
9 years, 14.56 between 10 and 14 years and 16.79 in 15 years of disease or more.

NM symptoms of PD are frequently missed during routine consultations. This fact may be related to that patients often do not associate these symptoms to PD or may be too embarrassed to discuss them. A recent study of Chaudhuri et al. ${ }^{5}$ showed that the most commonly omitted NM symptoms were delusions, daytime sleepiness, intense and vivid dreams, and dizziness.

A recent review by Soh et al. $^{6}$ identified three NMS among eight major determinants of quality of life in PD: depression, anxiety and fatigue. Furthermore, many NMS, as depression, restless legs, constipation, sleep disorders, daytime sleepiness and nocturia, are treatable.

\section{References}

1. Cosentino C, Nuñez Y, Torres L. Frequency of non-motor symptoms in Peruvian patients with Parkinson's disease. Arq Neuropsiquiatr 2013;71:216-219.

2. Chaudhuri KR, Martinez-Martin P, Schapira AHV, et al. An international multicenter pilot study of the first comprehensive self-completed nonmotor symptoms questionnaire for Parkinson's disease: the NMSQuest study. Mov Disord 2006;21:916-923.

3. Martinez-Martin P, Schapira AH, Stocchi F, et al. Prevalence of nonmotor symptoms in Parkinson's disease in an international setting; study using nonmotor symptoms questionnaire in 545 patients. Mov Disord 2007;22:1623-1629.
4. Barone P, Antonini A, Colosimo C. The PRIAMO study: a multicenter assessment of nonmotor symptoms and their impact on quality of life in Parkinson's disease. Mov Disord 2009;24:1641-1649.

5. Chaudhuri KR, Prieto-Jurcynska C, Naidu Y, et al. The nondeclaration of nonmotor symptoms of Parkinson's disease to health care professionals: an international study using the nonmotor symptoms questionnaire. Mov Disord 2010;25:704-709.

6. Soh SE, Morris ME, McGinley JL. Determinants of health-related quality of life in Parkinson's disease: a systematic review. Parkinsonism Relat Disord 2011;17:1-9. 